\title{
Strafschärfungen für Rückfalltaten: Ein Rückfall des vorgewarnten Gesetzgebers?
}

\section{Die Ausgangssituation}

Bis zum Ende der 1960er Jahre enthielt das bundesdeutsche Strafgesetzbuch vier Einzelfälle von Strafschärfungen für Rückfalltaten. Genügte beim Raub eine einschlägige Vorverurteilung ( $\$ 250$ Abs. 1 Nr. 5 StGB), verlangten die Bestimmungen über die Rückfallschärfung beim Diebstahl ( $\$ 244$ f. StGB), bei der Hehlerei ( $\$ 261$ StGB) und beim Betrug ( $\$ 264$ StGB) jeweils zwei gleichwertige (Diebstahl) bzw. gleichartige Vorverurteilungen (Hehlerei, Betrug). In Fortführung der in den jeweiligen Grundtatbeständen damals vorgesehenen ausschließlich stationären Rechtsfolgenbestimmungen legten die speziellen Rückfallvorschriften zudem fest, dass die voraufgegangenen Gefängnis- bzw. Zuchthausstrafen mindestens z. T. verbüßt bzw. erlassen sein mussten, um bei erneuter Verurteilung eine Strafschärfung begründen zu können. ${ }^{1}$ Mit der Streichung der besonderen Rückfallregelungen durch das 1. StrRG im Jahre 1969 nahm der Gesetzgeber zutreffende Kritik an strafschärfenden Rückfallbestimmungen auf, die auf ein bloß formelles Kriterium, das der Vorverurteilungen, abstellten.

Sein Rückzug war jedoch bestenfalls halbherzig. Denn der Gesetzgeber fügte $\S 17$ StGB bzw. dessen Nachfolgebestimmung $(\S 48 \mathrm{StGB})^{2}$ in den Allgemeinen Teil des StGB ein. ${ }^{3}$ Bei diesen grundsätzlich für alle Delikte geltenden Rückfallvorschriften handelte es sich um materielle. Als materielles Erfordernis diente das in der neuen vorsätzlichen Straftat zum Ausdruck gekommene Hinwegsetzen über die Warnfunktion der Vorverurteilungen (»daß er sich die früheren Verurteilungen nicht hat zur Warnung dienen lassen «). ${ }^{4}$ In einem solchen Verhalten sahen Befürworter der Rückfallklausel verstärkte kriminelle Energie am Werk, die eine erhöhte Tatschuld begründen und damit die Strafschärfung legitimieren sollte. ${ }^{5}$ Begleitet wurde die materielle Rückfallklausel von zwei formellen Erfordernissen, nämlich zwei Vorverurteilungen und einer Vorverbüßung von mindestens dreimonatiger Freiheitsstrafe. Die gesetzlich vorgesehene Folge bei einem derartigen Rückfall war eine mit Ausnahme der Ahndung von Bagatellunrecht - der in der Praxis allerdings häufigste Anwendungsfall moderate Belastung des rückfälligen Delinquenten. Die $\S \S 17$ und 48 StGB hoben das Mindeststrafmaß auf sechs Monaten Freiheitsstrafe. ${ }^{6}$ Für etliche Delikte, darunter die

1 Hanack, S. 100 (101), in: Jürgen Baumann, Programm für ein neues Strafgesetzbuch (1968).

2 Sturm, MDR 1979, S. 369.

3 Die Gestaltung der Rückfallbestimmungen zeichnet das BVerfG E 50, 125 (126) knapp nach; zur Forderung nach Wegfall des § 17 StGB: Mir Puig, ZStW 86 (1974), S. 195 (208).

4 Stree, in: Schönke/Schröder, StGB, 22. Aufl. (1985), § 48 Rn 17, zur individuellen Feststellung der Missachtung des Warnappells; Mir Puig, ZStW 86 (1974), S. 175 Fn 2.

5 BVerfGE 50, 125 (134 f.); Horstkotte, JZ 1970, S. 152 (153); Sturm, MDR 1979, S. 369 (370); Dölling, NJW 1987, S. 1041 (1045) referierend.

6 Horstkotte, JZ 1970, S. 152; Stree, in: Schönke/Schröder, 22. Aufl. (1985), § 48 Rn 20. 
damalige Unzucht mit Kindern, ${ }^{7}$ erwiesen sich die beiden in ihren Anforderungen übereinstimmenden ${ }^{8}$ Rückfallvorschriften jedoch als unnötig, weil deren Mindeststrafrahmen sechs Monate oder höher lag. ${ }^{9}$ Kritiker werteten daher die allgemeinen Rückfallbestimmungen als irrationale Trotzreaktion, als Ausdruck der Enttäuschung über frustrane Vorverurteilungen und sahen in ihnen einen pauschalen Ungehorsamszuschlag. ${ }^{10}$ Insbesondere bezweifelte man, dass Vorverurteilungen Hemmungsimpulse aufbauen könnten. ${ }^{11}$

Mit dem 23. StÄG aus dem Jahre 1986 hob der Gesetzgeber schließlich § 48 StGB auf. ${ }^{12}$ Die weithin einmütig zustimmende Resonanz des Schrifttums fand eine unterschiedliche Abstufung von gemessenem Beifall ${ }^{13}$ bis $\mathrm{zu}$ nahezu begeistertem $\mathrm{Zu}$ spruch $^{14}$. Nur vereinzelt wurde eine Strategie zur Bekämpfung des Rückfalls angemahnt. ${ }^{15}$

Für gut ein Jahrzehnt spielte das Thema von allgemeinen oder deliktsspezifischen Rückfallnormierungen in der bundesdeutschen Kriminalpolitik keine Rolle, bevor im Jahre 1998 mit $§ 176$ a Abs. 1 Nr. 4 StGB als einer Variante des schweren sexuellen Missbrauchs von Kindern die Rückkehr eines überwunden geglaubten Instruments die Fachwelt überraschte. Da Rückfalluntersuchungen nach Vollzug stationärer Rechtsfolgen regelmäßig einen Untersuchungszeitraum von fünf Jahren nach Entlassung zugrundelegen, ${ }^{16}$ ist nun der Zeitpunkt gekommen, die inzwischen als $\S 176$ a Abs. 1 StGB firmierende Variante einer genaueren Analyse zu unterziehen.

Nach Behandlung von Entstehungsgeschichte (II.) stehen Normstruktur (III.) und die Untersuchung der Verfassungsmäßigkeit des § 176 a Abs. 1 StGB im Zentrum (IV.), bevor eine Erwartung an künftiges Gesetzgebungsverhalten formuliert werden soll (V.).

\section{Die Entstehungsgeschichte der Rückfallqualifikation in $\$ 176$ a Abs. 1 StGB}

Das Gesetzgebungsverfahren zeigt den Kompromisscharakter der Rückfallbestimmung in $\S 176$ a Abs. 1 StGB klar auf. Bestrebungen, den sexuellen Missbrauch von Kindern gem. $\S 176$ StGB zum Verbrechenstatbestand umzugestalten, fanden keine ausreichende Zustimmung. Um das Gesamtpaket des 6. StrRG mit Mehrheit beschließen zu können, wurde sodann die spezifische Rückfallbestimmung in $\S 176$ a Abs. 1

7 Horstkotte, JZ 1970, S. 152.

8 Sturm, JZ 1979, S. 369.

9 Berckhauer, MschrKrim 1982, S. 270 (277); Meier, ZStW 95 (1983), S. 316 (338 f.).

10 Jung, JuS 1986, S. 741 (743).

11 Meier, ZStW 95 (1983), S. 316 (317); Geiter, ZRP 1988, S. 376 (378).

12 Renzikowski, NStZ 1999, S. 440 (441); Erhard, Strafzumessung bei Vorbestraften unter dem Gesichtspunkt der Strafzumessungsschuld, Berlin 1992, S. 41.

13 Dölling, NJW 1987, S. 1041 (1045).

14 Jung, JuS 1986, S. 741 (743); Geiter, ZRP 1988, S. 376 (381).

15 Zipf, in: FS f. Herbert Tröndle (1989), S. 439 (442).

16 Siehe etwa Berckhauer, MschrKrim 1982, S. 270 (274); vgl. Kerner, in: KKW, 3. Aufl. (1993), S. 432 (433); Jörg-Martin Jehle/Wolfgang Heinz/Peter Sutterer, Legalbewährung nach strafrechtlichen Sanktionen, Berlin 2003, S. 7 (vierjähriger Rückfallzeitraum). 
Nr. 4 StGB aufgenommen. Diese Normierung war das Zugeständnis an die Anhänger der Verbrechenslösung. ${ }^{17}$

Im Unterschied zu den anderen Varianten des schweren sexuellen Missbrauchs, die nun im Mindestmaß mit einer Strafdrohung von zwei Jahren Freiheitsstrafe bedroht sind und in den Absatz 2 des $§ 176$ a StGB rückten, hat das Gesetz zur Änderung der Vorschriften über die Straftaten gegen die sexuelle Selbstbestimmung pp. vom 27.12.2003 den Verbrechenstatbestand des gleichartigen Rückfalls nach Nr. 4, ohne eine inhaltliche Veränderung vorzunehmen, als $\S 176$ a Abs. 1 StGB an die Spitze der Vorschrift gestellt. ${ }^{18}$

\section{Die Normstruktur des $§ 176$ a Abs. 1 StGB}

Die Qualifikation als Verbrechenstatbestand erfordert eine einschlägige Vorverurteilung wegen sexuellen Missbrauchs gem. $§ 176$ Abs. 1 und Abs. 2 StGB. ${ }^{19}$ Das Gesetz setzt mit der Bedingung der gleichartigen Vorverurteilung also nur ein formelles Erfordernis voraus. Im Unterschied zu $\S 48$ StGB a. F. verzichtet der Normtext auf die Missachtung des Warnappells der Vorverurteilung und begnügt sich mit der schematischen Handhabung des einschlägigen Rückfalls. Umstritten ist, ob eine verfassungskonforme Auslegung des $\S 176$ a Abs. 1 StGB gebietet, dem jeweiligen Täter konkret den Vorwurf zu machen, die aus einer früheren Verurteilung erfolgte Warnung in den Wind geschlagen zu haben. ${ }^{20}$ Bevor die Verfassungsmäßigkeit des $\S 176$ a Abs. 1 StGB analysiert wird, soll zunächst die Sachangemessenheit der Aufstufung zum Verbrechen Thema sein.

Fraglich ist, ob die gleichartige Vorverurteilung die Einstufung eines sexuellen Missbrauchs von Kindern als Verbrechen wegen erhöhten Unrechts und/oder erhöhter Schuld zu rechtfertigen vermag. Denn maßgeblich für die Abgrenzung von Verbrechen und Vergehen ist anerkanntermaßen die quantitative Abstufung nach dem Schweregrad der strafbaren Handlungen. ${ }^{21}$ Für Straftaten mit im Unterschied zu Vergehen höherem Unwertgehalt steht in Verfolgung des Verhältnismäßigkeitsprinzips die Deliktskategorie der Verbrechen zur Verfügung. ${ }^{22}$

17 Kreß, NJW 1998, S. 633 (638 f.); Lenckner/Perron, 26. Aufl. (2001), § 176 a Rn 7; Renzikowski, NStZ 1999, S. 440 (441).

18 BGBl. I 2003, S. 3007; Duttge/Hörnle/Renzikowski, NJW 2004, S. 1065 (1066).

19 BGH NStZ 2002, 198 (199).

20 BGH NStZ 2002, 198 (199); Lenckner/Perron, in: Schönke/Schröder, 26. Aufl. (2001), $\S 176$ a Rn 7.

21 Baumann/Weber/Mitsch, 11. Aufl. (2003), § 11 Rn 3; Eser, in: Schönke/Schröder, 26. Aufl. (2001), § 12 Rn 3; Stratenwerth, 4. Aufl. (2000), § 5 Rn 2; Zipf, in: FS f. Herbert Tröndle (1989), S. 439 (448).

22 LK-Gribbohm (1.3.1997), § 12 Rn 5; Cornelius Mirow, Neue Formen der Dichotomie der Straftaten, Berlin 2001, S. 128 ff. 


\section{1. Überlegungen zur Deliktsschwere von Rückfall allgemein}

a) Zweitdelikt als Gehorsamspflichtverletzung

Folgende These steht zur Diskussion: Die Tat eines Vorbestraften erhöhe das Handlungsunrecht wegen eines doppelten Pflichtenverstoßes. Nicht nur der Normbefehl als solcher werde missachtet, sondern in wiederholtem Normbruch liege auch eine Verletzung einer Gehorsamspflicht gegenüber dem Staat. ${ }^{23}$ Einer solchen Begründung kann freilich nicht gefolgt werden. Denn dem Bürger wird eine eigenständige Gehorsamspflicht durch die Strafrechtsordnung gerade nicht auferlegt. ${ }^{24}$ Eine Verurteilung kreiert keine Verhaltenserwartung an den Verurteilten zur allgemeinen Normbefolgung mit zusätzlicher Strafmöglichkeit über die bei einer strafbaren Handlung vorgesehene Rechtsfolge hinaus. Dies belegt zum einen der Rückschluss aus dem singulären § 145 a StGB, der den Verstoß gegen richterliche Weisungen während der Führungsaufsicht als spezifisches Unrecht unter Strafe stellt. ${ }^{25}$ Zum anderen ist die Wirkungslosigkeit eines Strafurteils zur Vermeidung erneuter Straffälligkeit nicht tatbestandsmäßig im Hinblick auf eine Strafvereitelung; denn Rückfall verhindert nicht die Bestrafung wegen einer strafbaren Handlung, ist also kein Delikt gegen die Strafrechtspflege. ${ }^{26}$

b) Zweitdelikt als spezifische Unrechtskomponente

Der erhöhte Unwert einer Rückfalltat könnte sich daraus ergeben, dass die wiederholte gleichartige Tat eine größere und beharrlichere Missachtung für das geschützte Rechtsgut bedeutet. ${ }^{27}$ Hiergegen ist freilich einzuwenden, dass mangelnde Akzeptanz des strafrechtlichen Schutzes bestimmter Rechtsgüter nicht die Integrität der Rechtsgüter als solche berührt, die strafrechtliche Haftung für die zweite strafbare Handlung bleibt unbenommen. ${ }^{28}$

\section{c) Zweitdelikt als Delikt mit erhöhtem Schuldvorwurf}

$\mathrm{Zu}$ prüfen ist, inwieweit eine Vorverurteilung eine besonders hohe Hemmschwelle vor der Begehung erneuter Straftaten errichtet und inwieweit deren Überwindung einen erhöhten Schuldvorwurf begründet. ${ }^{29}$ Hierbei sind normative und empirische Argumente zu trennen.

Das empirische Moment einer Abschreckungswirkung durch gerichtliche Verurteilung bzw. Vollstreckung von Freiheitsstrafe ist nicht einmal in Ansätzen belegt. ${ }^{30}$ Beachtliche Plausibilität kommt vielmehr der Überlegung zu, dass die mit erstmaliger Tatbegehung einhergehende Erweiterung des Handlungsrepertoires des Täters bestenfalls durch staatliche Antwort im verurteilenden Erkenntnis hierauf wiederum auf Al-

23 Vgl. Mir Puig, ZStW 86 (1974), S. 175 (189/191).

24 Tatjana Hörnle, Tatproportionale Strafzumessung, Berlin 1999, S. 162.

25 Pfeiffer, in: FS f. Günter Blau (1985), S. 291 (300).

26 Mir Puig, ZStW 86 (1974), S. 175 (191).

27 Mir Puig, ZStW 86 (1974), S. 175 (193/197 f.).

28 Mir Puig, ZStW 86 (1974), S. 175 (201 f.).

29 BVerfG E 50, 125 (136).

30 Eisenberg, 5. Aufl. (2000), § 31 Rn 55. 
ternativen eingeschränkt wird, die dem Strafrecht genügen. Es könnte im günstigsten Fall also ein vor erstmaliger Deliktsverwirklichung bestehendes Hemmungsniveau erreicht, keineswegs aber eine Verstärkung der Hemmschwelle im Vergleich mit allen Normunterworfenen aufgebaut werden. ${ }^{31}$ Nicht fernliegend ist sogar eine Entwicklung, dass die Wahlmöglichkeiten des potentiellen Täters im Hinblick auf Legalverhalten korrespondierend mit der Häufigkeit von Straftaten abnehmen. Die Verringerung sozialer Chancen infolge von Vorverurteilungen birgt das Risiko der Etablierung eines abweichenden Lebensstils. ${ }^{32}$ Hiernach müsste folgerichtig eine Reduktion des Schuldvorwurfs vorgenommen werden. ${ }^{33}$

Überdies bestehen Zweifel an der Warnfunktion der Vorverurteilung im Hinblick auf den maximalen Zeitraum von fünf Jahren zwischen Rückfalltat und voraufgegangenem Urteil. Wenn man einen Anruf zur Normbefolgung durch Urteil unterstellt, drängt sich die Frage auf, ob dieser überhaupt noch präsent ist, um handlungssteuernd zu wirken. ${ }^{34}$

In normativer Hinsicht verkennt die Hemmschwellenthese die Funktion der Strafbegründungsschuld. Sie legt die individuelle Verantwortlichkeit für ein bestimmtes Werk fest, ${ }^{35}$ ohne einen graduierbaren Schuldvorwurf zu erheben. ${ }^{36}$ Persönliche Verantwortung des Täters liegt vor, wenn er die Anforderungen des Rechts hätte erkennen und sich danach hätte verhalten können. Etwa erhöhte Normkenntnis, in Form von Bewusstsein der Strafnorm und Kenntnis der konkreten Strafwürdigkeit, ${ }^{37}$ ist unter Schuldgesichtspunkten irrelevant. ${ }^{38}$ Denn das qualitative gleiche Unrechtsbewusstsein kann auch in anderer Weise als durch eigene Vorverurteilung erworben worden sein, mithin nicht die Ungleichbehandlung von Rückfalltäter mit Vorverurteilung und Ersttäter ohne Vorverurteilung aber mit identischem Unrechtsbewusstsein erklären. ${ }^{39}$

\section{d) Zweitdelikt als Gebot erhöhter Normbestätigung}

Teils versteht das Schrifttum den strafrechtlichen Schuldbegriff funktional i. S. d. Integrationsprävention. An die Stelle der Vorwerfbarkeit rückt die Frage, ob der anerkannte Strafzweck es gebietet, den Täter zur Verantwortung zu ziehen, oder eine solche Form der Zuschreibung unterbleiben kann. Präventive Erfordernisse in der Art, dass Rückfall intensivierte Normbestätigungsbedürfnisse auslöse, denen mit einer Verbrechenseinstufung und dem erhöhten Strafrahmen Rechnung getragen werde, ${ }^{40}$

31 Erhard, S. 68 f.

32 Pfeiffer, in: FS f. Günter Blau (1985), S. 291 (301); Edwin M. Lemert, Social Pathology, New York u. a. 1951, S. 77.

33 Eisenberg, 5. Aufl. (2000), § 31 Rn 54; Mir Puig, ZStW 86 (1974), S. 175 (202/206).

34 Pfeiffer, in: FS f. Günter Blau (1985), S. 291 (301); Schneider, Jura 1996, S. 337 (338).

35 Kühl, 4. Aufl. (2002), § 10 Rn 2; Roxin, AT I, (1992), § 19 Rn 46, Stratenwerth, 4. Aufl. (2000), § $10 \mathrm{Rn} 4$.

36 Hörnle, S. 161; Mir Puig, ZStW 86 (1974), S. 175 (197); a. A.: Jescheck/Weigend, 5. Aufl. (1996), S. 426 unter Bezugnahme auf das Ausmaß von Rechtsgesinnung als Schuldelement.

37 Mir Puig, ZStW 86 (1974), S. 175 (185).

38 Frisch, ZStW 99 (1987), S. 750 (774).

39 Mir Puig, ZStW 86 (1974), S. 175 (185).

40 NK-Frommel (30.11.2001), § 176 a Rn 10, wonach die Strafschärfung aus spezial- und generalpräventiven Gründen angemessen sei - ohne Begründung. 
lassen sich jedoch nicht mit dem Anspruch einer individuellen Sanktionsnotwendigkeit vereinbaren. ${ }^{41}$

e) Zusammenfassung

Wiederholte Straftatbegehung begründet also im Allgemeinen keinen Unwert, der es rechtfertigt, die erneute strafbare Handlung zu einem Verbrechen aufzustufen. Es wäre zudem ein Wertungswiderspruch, den Rückfall im Vergleich zu mehreren real konkurrierenden Straftaten bzw. zur fortgesetzten Begehung etlicher Delikte wie z. B. in $\S 244$ Abs. 1 Nr. 2 StGB als schwerere Deliktskategorie zu behandeln.

\section{2. Überlegungen zur Deliktsschwere wiederholten sexuellen Missbrauchs}

Auch bei gleichartigem Rückfall wegen sexuellen Missbrauchs von Kindern muss diese Straftat im Unterschied zum erstmaligen Missbrauch einen erhöhten Unwert aufweisen, soll die Qualifizierung als Verbrechen einfachrechtlich keine Bedenken hervorrufen. Auf dem Boden eines materiellen Verständnisses von Rückfall mit dem Kriterium der Warnfunktion infolge Vorverurteilung ${ }^{42}$ stellt sich mithin folgende Frage: Verfügen Täter eines sexuellen Missbrauchs im Allgemeinen sowohl über das intellektuelle Moment, den mit einer Verurteilung verbundenen Anruf zur Normkonformität zu begreifen, als auch über die voluntative Kompetenz, sich entsprechend dem Normappell zukünftig zu verhalten ? $^{43}$

Im Kontext der materiellen Rückfallklausel zu $\S 17 \mathrm{StGB}$ a. F. finden sich in den 1970er Jahren zu damals sog. Triebtätern die Bedenken, dass diese Tätergruppe Defizite in den angesprochenen Kompetenzbereichen ausweist. Bei Auslieferung an eine unglückliche Veranlagung könne die Triebstärke die Warnfunktion früherer Verurteilungen überdecken. ${ }^{44}$ Zumindest zeigt diese Auffassung an, dass eine typisierende Annahme erhöhten Schuldvorwurfs auch bei Straftaten des sexuellen Missbrauchs fehl gehen dürfte. Für Zipf hat das Begehen von Straftaten infolge eines Hangs, dargestellt am Beispiel von Exhibitionisten, sogar gegenteilig eine Reduktion des Schuldvorwurfs zur Folge. ${ }^{45}$

In Fällen verminderter Schuldfähigkeit dürften Einsichts- und Steuerungsfähigkeit zumindest wesentlich beeinträchtigt sein. ${ }^{46}$ Ein solcher Fakt verhindert, dass ein Warnappell, einmal angenommen eine Vorverurteilung enthalte einen solchen, den Täter wirksam erreicht.

41 Hörnle, S. 164.

42 Während Renzikowski, NStZ 1999, S. 440 (441), § 176 a StGB im Wege verfassungskonformer Auslegung auf die Einzelfallfeststellung erhöhter Schuld festlegen will, schlagen SK-Horn/Wolters (September 2002), § 176 a Rn 3 i, eine Überprüfung des $§ 176$ a StGB im Wege der konkreten Normkontrollklage nach Art. 100 Abs. 1 GG vor.

43 Vgl. OLG Hamburg NStZ 1983, 366; OLG Köln StV 1982, 228.

44 OLG Stuttgart MDR 1974, 685; Hartmut Frosch, Die allgemeine Rückfallvorschrift des $§ 48$ StGB, Tübingen 1976, S. 75; Stree, in: Schönke/Schröder, 22. Aufl. (1985), § 48 Rn 17 a. E.

45 Zipf, in: FS f. Herbert Tröndle (1989), S. 439 (444), damit seine Position in Maurach/Gössel/Zipf, 6. Aufl. (1984), § 64 Rn 40 aufgebend.

46 OLG Köln StV 1982, 228. 
Konkretisiert auf sexuellen Missbrauch von Kindern ist anzuführen, dass eine beachtliche Zahl von Tätern nicht über ungeschränkte Schuldfähigkeit verfügt. Nach $\mathrm{Elz}$ schlossen die Gerichte bei mehr als einem Fünftel der untersuchten Täter im Verurteilungszeitraum 1987 eine verminderte Schuldfähigkeit nicht aus. ${ }^{47}$ Bei Verurteilungen von rückfälligen Sexualstraftätern ermittelt Elz sogar in mehr als einem Drittel der Fälle verminderte Schuldfähigkeit. Mit diesem Befund kompatibel ist das Datum, dass einschlägig Rückfällige signifikant seltener wegen schweren sexuellen Missbrauchs von Kindern nach $\S 176$ Abs. 3 StGB a. F. verurteilt wurden als solche Sexualstraftäter, die erstmals (wegen schweren sexuellen Missbrauchs) verurteilt wurden. ${ }^{48}$ Die jüngsten zur Verfügung stehenden Daten der Strafverfolgungsstatistik, die freilich keine Differenzierung nach den einzelnen Varianten des $\S 176$ a StGB a. F. vornehmen, bestätigen das von Elz ermittelte Muster eines mit der Schwere des sexuellen Missbrauch zunehmenden Anteils vermindert Schuldfähiger. So kam im Jahre 2001 $\S 21$ StGB bei $8,1 \%(n=83)$ der 1.027 nach allgemeinem Strafrecht verurteilten Täter wegen sexuellen Missbrauchs nach $\S 176 \mathrm{StGB}^{49}$, hingegen bei 14,8\% $(n=60)$ der 405 nach $\S 176$ a StGB Verurteilten ${ }^{50}$ zur Anwendung. Im Jahr zuvor beliefen sich die entsprechenden Raten auf 10,5\% bei Verurteilungen wegen $\S 176$ StGB ( $n=127)$ bzw. $14 \%$ bei Verurteilungen nach $\S 176$ a StGB $(n=52) .^{51}$

Angesichts der nennenswerten Beschränkungen und insbesondere dem höheren Anteil vermindert Schuldfähiger bei Verurteilungen wegen schweren sexuellen Missbrauchs unterstellt die typisierte Betrachtung in $\S 176$ a Abs. 1 StGB - abweichend von den vorgefundenen Täterprofilen - nur erhöhte Schuld. Der Rückfall ist mithin kein sachgerechter Erschwerungs- und Qualifizierungsgrund. ${ }^{52}$ Eine Verbrechenseinstufung lässt sich so nicht rechtfertigen.

\section{Fazit}

Im Ergebnis führt das Vorverurteilungskriterium zu keiner sachlichen Begründung der Verbrechensqualität: Wiederholte einschlägige Tatbegehung beinhaltet keine vom Grundtatbestand des $§ 176$ Abs. 1 u. 2 StGB abweichende Schweredimension.

Eine inhaltlich unzureichende Strafnorm verfällt aber nicht ohne weiteres der Verfassungswidrigkeit, - insbesondere räumt das BVerfG dem Gesetzgeber einen weiten Gestaltungsspielraum zur Bestimmung der Strafrahmen ein,$-{ }^{53}$ so dass $\S 176$ a Abs. 1 StGB einer eigenständigen verfassungsrechtlichen Überprüfung zu unterziehen ist.

47 Jutta Elz, Legalbewährung und kriminelle Karrieren von Sexualstraftätern, Wiesbaden 2001, S. 173.

48 Elz, S. 247.

49 Strafverfolgung 2001, Vollständiger Nachweis der einzelnen Straftaten, Wiesbaden 2003, S. 281 mit S. 74, eigene Berechung.

50 Strafverfolgung 2001, Vollständiger Nachweis der einzelnen Straftaten, Wiesbaden 2003, S. 281 mit S. 74, eigene Berechung.

51 Strafverfolgung 2000, Vollständiger Nachweis der einzelnen Straftaten, Wiesbaden 2001, S. 279 mit S. 72, eigene Berechung.

52 Nelles, Anhörung vor dem Rechtsausschuss des Dt. Bundestags vom 19.2.2003, S. 41.

53 BVerfG E 50, 125 (138/140); BVerfG NJW 1994, 1577 (1579) - Cannabis-Beschluss zum gegenständlichen Bereich strafbaren Handelns. 
IV. Verfassungsmäßigkeit des $\$ 176$ a Abs. 1 StGB?

Für den BGH steht die Verfassungsmäßigkeit des $\S 176$ a Abs. 1 Nr. 4 StGB a. F. außer Frage. ${ }^{54}$ Daher unterlässt der BGH eine Auseinandersetzung mit den Kriterien der Übereinstimmung einer Strafnorm mit den Bestimmungen des Grundgesetzes, den ungeschriebenen Verfassungsgrundsätzen und den Grundentscheidungen des Grundgesetzes. ${ }^{55}$ Bezogen auf das Tatschuldprinzip erfolgt nun eine kurze verfassungsrechtliche Würdigung.

\section{Tatschuldprinzip}

Das Tatschuldprinzip besagt, dass Strafe Schuld voraussetzt. Das Tatschuldprinzip hat nach ständiger verfassungsgerichtlicher Rechtsprechung Verfassungsrang. Seine Grundlage findet es im Rechtsstaatsprinzip und in Art. 1 Abs. 1 GG. ${ }^{56}$ Zwei Komponenten des Tatschuldprinzips sind zu unterscheiden.

a) Entsprechung von Rechtsvoraussetzungs- und Rechtsfolgenseite

Im Hinblick auf eine angehobene, angedrohte Strafe bedeutet das Tatschuldprinzip, dass es typischerweise erhöhte Schuld voraussetzt und die verschärfte Sanktion dem Maß vermehrter Schuld entsprechen muss. ${ }^{57}$ Eine Strafschärfung wegen Rückfalls setzt daher einen erhöhten Schuldvorwurf voraus. M. a. W.: Erhöhte Schuld als Korrektiv der Rückfallstrafschärfung ist unabdingbar. Die materiell-rechtliche Rückfallklausel mit dem Erfordernis einer Einzelfallprüfung des $\S 48$ StGB bestand die verfassungsgerichtliche Kontrolle; sie soll mit dem verfassungsrechtlichen Schuldgrundsatz vereinbar sein. ${ }^{58}$ Dem herausgehobenen Stellenwert individueller Schuldfeststellung wird bei einer formellen Rückfallklausel, wie sie $\S 176$ a Abs. 1 StGB enthält, nicht genügend Rechnung getragen: der notwendige, die Strafmaßanhebung rechfertigenden Konnex findet sich nicht im Normtext. In $\S 176$ a Abs. 1 StGB fehlt nicht nur die für den ungleichartigen Rückfall, sondern auch die bei gleichartigem Rückfall vorausgesetzte konkrete Feststellung erhöhter Schuld. ${ }^{59}$

b) Gegenstand der Schuld

Bezugsgegenstand der Tatschuld ist die jeweilige einzelne Tathandlung als solche und nicht abgeurteilte Umstände der Vergangenheit oder sonstige Fehlhaltungen. ${ }^{60}$ Dem Täter wird die Willensbildung in Bezug auf die begangene Tat zum Vorwurf ge-

54 BGH NStZ 2002, 198 (199).

55 Vgl. BVerfG NJW 1994, 1577 (1579) - Cannabis-Beschluss.

56 BVerfG E 50, 125 (133); 45, 187 (228); 20, 323 (331).

57 BVerfG E 50, 125 (134); Hillenkamp, GA 1974, S. 208 (213).

58 BVerfG E 50, 125 (136 f.); 36, 41 (46); auch wenn Maurach/Gössel/Zipf, 6. Aufl. (1984), $\S 64 \mathrm{Rn} 27$ Bedenken äußern, sehen sie noch eine Vereinbarkeit des § 48 StGB mit dem Grundgesetz.

59 BVerfGE 50, 125 (136) u. (137 - zum ungleichartigen Rückfall); OLG Köln StV 1982, 228; Frosch, S. 70.

60 Hanack, S. 100 (110). 
macht. ${ }^{61}$ Eine voraufgegangene Verurteilung nimmt keinen Einfluss auf Erfolgs- oder Handlungsunrecht der nunmehr angeklagten Normverletzung: ${ }^{62}$... »eine direkte Relevanz der Vortat [ist] in einem Tatschuldstrafrecht ein Widerspruch in sich « ${ }^{63}$

Hiernach dürfte $\S 176$ a StGB schon wegen Unvereinbarkeit mit dem Tatschuldprinzip einer verfassungsrechtlichen Überprüfung nicht standhalten.

\section{Ausblick auf zukünftige gesetzgeberische Initiativen}

Selbst wenn die Rückfallklausel in § 176 a Abs. 1 StGB Ausdruck populistischen Denkens ist ${ }^{64}$ kann hieraus eine sachangemessene Begründung nicht abgleitet werden. Denn Strafrechtsnormen müssen wertrationalen Erwägungen, zu denen der Schuldgrundsatz, der Verhältnismäßigkeitsgrundsatz und die Rechtsstaatlichkeit des Strafverfahrens zählen, ${ }^{65}$ folgen. Solche fundieren - wie aufgezeigt - allerdings den Verbrechenstatbestand bei gleichartigem Rückfall des sexuellen Missbrauchs von Kindern nicht. Der Gesetzgeber kannte die von ihm sog. Vorwerfbarkeitsklausel in § $48 \mathrm{StGB}$ ebenso wie die rechtstatsächliche Entbehrlichkeit einer allgemeinen Rückfallbestimmung. ${ }^{66}$ Er war also vorgewarnt. Mit der Schaffung einer nicht verfassungsfesten Norm steht der Gesetzgeber daher in der Pflicht, gleichsam als Akt notwendiger und tätiger Reue, § 176 a Abs. 1 StGB zu streichen.

Für eine kleine besonders gefährliche Klientel von Sexualstraftätern, die ihre Opfer unter Kindern finden, sind das Maßregelrecht und außerstrafrechtliche Interventionen der richtige Weg; bei den übrigen gibt § $46 \mathrm{Abs}$. $2 \mathrm{StGB}$ das geeignete Instrument zur Festsetzung einer angemessenen Strafe für wiederholten sexuellen Missbrauch von Kindern.

61 Jescheck/Weigend, 5. Aufl. (1996), S. 429; Frosch, S. 59.

62 Frisch, ZStW 99 (1987), S. 750 (773).

63 Frisch, ZStW 99 (1987), S. 750 (774); Jescheck/Weigend, 5. Aufl. (1996), S. 23.

64 Duttge/Hörnle/Renzikowski, NJW 2004, S. 1065 (1067).

65 Jescheck/Weigend, 5. Aufl. (1996), S. 22 f.; Kaiser, 3. Aufl. (1996), § 99 Rn 4.

66 BR-Drs. 370/84, S. 1 (10). 